\title{
Reflection and Transmission of Gamma Radiation by Barriers: Semianalytic Monte Carlo Calculation ${ }^{1}$
}

\author{
Martin J. Berger and John Doggett
}

\begin{abstract}
The transport equation for photons which have been Compton-scattered repeatedly with a specified sequence of energy losses and deflections was solved analytically, by an "orders of scattering" approach, to obtain conditional reflection and transmission probabilities. These probabilities were then averaged over all possible intermediate angular and energy configurations in a Monte Carlo calculation carried out on the NBS automatic computer (SEAC). The efficiency of the Monte Carlo calculation was increased by taking advantage of the close correlation between the transmission of radiation through thick barriers and the corresponding penetration problem in an infinite homogeneous medium for which an exact solution was available. The reflected and transmitted energy flux (integrated over all spectral energies and directions) has been calculated for $0.66-, 1-$ and $4-\mathrm{Mev}$ radiation incident on water barriers, and 1-, 4-, and 10-Mev radiation incident on iron, tin, and lead barriers. Angular and energy spectra were obtained for water at $0.66 \mathrm{Mev}$. The ratio of the scattered energy flux, transmitted through a barrier to the scattered flux at equal depth in an infinite medium, was found to be constant for distances from the source greater than four mean free paths of the source radiation.
\end{abstract}

\section{Introduction}

The theory of the diffusion and penetration of gamma radiation in an infinite homogeneous medium is now well developed, complete solutions being provided by the moment-method [1] ${ }^{2}$ and the closely related semiasymptotic Laplace-transform method [2]. A satisfactory theory of boundary effects is still lacking. The "orders of scattering" approach [3] provides formal solutions to boundary problems, which can in practice be evaluated only when few orders of scattering need be considered. The Monte Carlo method [4] is a very effective means for treating boundary effects but is inefficient when applied to the radiation that penetrates very far from the source. This inefficiency can, in principle, be removed by so-called "biased" sampling, but the success of such techniques has not yet been demonstrated conclusively.

This investigation is part of an exploratory Monte Carlo program for the survey of boundary effects in gamma-ray diffusion. The specific aim is to calculate the reflection and transmission of radiation by plane parallel barriers. The approach is eclectic, and a combination of all three of the methods described above is being used. It can be characterized as an "orders of scattering" method in which the multiple integrals, which ordinarily make the evaluation of the solution troublesome, are evaluated numerically by random sampling. Conversely, one could call it a "reinforced" Monte Carlo calculation whose efficiency for dealing with deep penetration has been increased in two ways: by confining the random sampling to the angular and energy variables, while treating the space variable analytically, and through the use of correlated sampling, the correlated problem being the diffusion of $\gamma$-radiation in an infinite homogeneous medium.

\footnotetext{
This work was supported by the Office of Naval Research and the Reactor Division of the U.S. Atomic Energy Commission.

2 Figures in brackets indicate the literature references at the end of this paper
}

The reflection and transmission problems differ substantially from the point of view of random sampling, because transmission through thick barriers, being unlikely, tends to require an excessive amount of sampling and thus puts a high premium on analytical help. Nevertheless, the two problems were treated together because they fitted in a single computational scheme.

Two types of questions may be asked in radiation diffusion problems. One concerns the estimation of the radiation intensity at any one position, corresponding to the observable response of some integral detector like an ionization chamber. The other type of question is more detailed and concerns the spectral composition and directional distribution of the radiation at any one point. In the work reported here most of the effort has been devoted to intensity (energy flux) determinations. Exploratory studies of spectral and angular distributions were made only for one source energy $(0.66 \mathrm{Mev})$ and one barrier material (water).

For the deep-penetration problem the attention was focused on the comparison of the radiation intensity behind a finite barrier of thickness $t$ and inside an infinite medium at a depth $z$ (equal to $t$ ) from the source plane (see fig. 1,a,b). No quantitative indication was previously available on this comparison. Notice that practical situations are usually intermediate between those schematized in figure $1, a, b$, because there is some matter (possibly of different composition) behind the barrier, though often at some distance from it (fig. 1,c).

On the problem of relative intensity a rather definitive answer has been obtained. In the first place, it proved unnecessary to have extended the calculation to very great depths because the intensity ratio no longer varies appreciably beyond about four mean free paths of the incident radiation. It follows that any future investigation may confine itself to moderate depths and may thus utilize less elaborate methods. Data on the values of the 

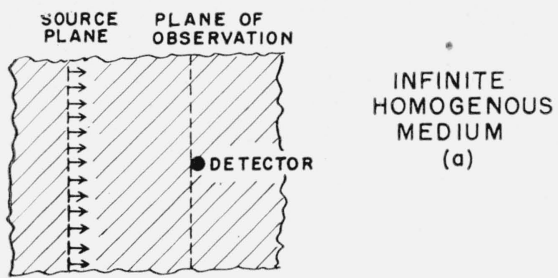

(a)

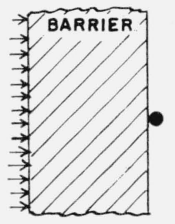

IDEALIZED

BARRIER

PROBLEM

(b)
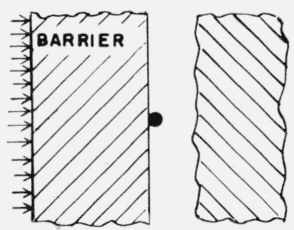

ACTUAL SITUATION

(c)
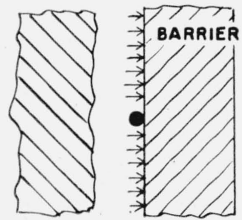

Figure 1. Schematization of the barrier reflection-transmission problem.

intensity ratio for various depths are given in table 3 for four materials (water, iron, tin, and lead) and four $\gamma$-ray energies $(0.66,1,4$, and $10 \mathrm{Mev})$. Those data may be applied as correction factors to the extensive results of calculations for infinite media [5]. The ratios differ substantially from unity only for low energies and low- $Z$ materials, and also vary smoothly, so that adaptation to other circumstances ought to be easy.

In the treatment of the reflection problem, attention was directed in the first place to the dependence of the reflected intensity on the $\gamma$-ray energy, on the material of the barrier, and also on the angle of incidence. The results for a semi-infinite barrier are given in table 2,a. An additional item of interest was the dependence of reflection on the barrier thickness, that is, on how rapidly the reflection approaches the value for a semi-infinite barrier. Some data on this aspect are given in table 2,b.

\section{Outline of the Calculation}

\subsection{Analytical Frame}

We consider a homogeneous plane-parallel layer of material located between the planes $z=0$ and $z=t$. Radiation is incident with specified energy and direction on the face $z=0$. What are the characteristics of the radiation reflected through the plane $z=0$ and transmitted through the plane $z=t$ ?

The state of a photon traversing the barrier is described by three variables: the energy $E$, the angle $\theta$ between the direction of motion and the $z$-axis, and the depth $z$ in the barrier.

Photons can be scattered and absorbed inside the barrier. Let $\mu_{A}(E)$ equal the probability of absorption per unit path length; $c(E)$ the probability of scattering per unit path length, and $\Psi\left(E, \theta ; E^{\prime}, \theta^{\prime}\right)$ the probability distribution (normalized to unity) that a scattering will change the state of the photon from $(E, \theta)$ to $\left(E^{\prime}, \theta^{\prime}\right)$. Consider a photon that, as the result of $n$ scatterings, has successively assumed the energies and directions

$$
\left\{\begin{array}{ccc}
E_{0}, E_{1}, \ldots, E_{n} \\
\theta_{0}, \theta_{1}, \ldots & ., \theta_{n}
\end{array}\right\}=\text { energy-angle history } h_{n} .
$$

The index $n$ refers to the state of the photon immediately after its $n$th scattering; $n=0$ pertains to the state in which the photon enters the barrier. The probability distribution of $h_{n}$ is

$$
Q_{n}=\prod_{m=0}^{n-1} \Psi\left(E_{m}, \theta_{m} ; E_{m+1}, \theta_{m+1}\right)
$$

Let $P_{n}\left(z, t, h_{n}\right)$ be the probability that a photon with history $h_{n}$ will cross the plane $z(0 \leq z \leq t)$ after the $n$th and prior to an $(n+1)$ st scattering. An analytical expression for this crossing-probability is derived in section 3 . If a beam of $K$ photons per unit area and unit time is incident on the barrier, the flux per unit time at depth $z$ is

$$
\begin{aligned}
F(E, \theta, z, t)=K \sum_{n=0}^{\infty} f_{n} \int d E_{1} \int d \theta_{1} \ldots \int d E_{n} \\
\\
\qquad d \theta_{n} P_{n} Q_{n} \delta\left(E-E_{n}\right) \delta\left(\theta-\theta_{n}\right),
\end{aligned}
$$

where $\delta$ is Dirac's delta function. If the weight factors $f_{n}$ are unity, the flux represents the number of photons crossing a unit area of the z-plane per unit time. Most radiation detectors do not measure the number flux through a fixed plane; their response

\begin{tabular}{|c|c|c|c|}
\hline Type & $f_{n}$ & & Flux \\
\hline $\begin{array}{l}\mathrm{a} \\
\mathrm{b}\end{array}$ & $\begin{array}{c}1 \\
E_{n}\end{array}$ & $\begin{array}{l}\text { Number } \\
\text { Energy }\end{array}$ & $\left\{\begin{array}{l}\text { Flux (or current) per unit } \\
\text { area of surface perpen- } \\
\text { dicular to the } z \text {-axis. }\end{array}\right.$ \\
\hline $\begin{array}{l}\mathrm{c} \\
\mathrm{d}\end{array}$ & $\begin{array}{c}\left|\sec \theta_{n}\right| \\
\left|\sec \theta_{n}\right| E_{n}\end{array}$ & $\begin{array}{l}\text { Number } \\
\text { Energy }\end{array}$ & $\begin{array}{l}\text { Flux per unit area of sur- } \\
\text { face perpendicular to the } \\
\text { direction of motion of the } \\
\text { radiation. This is the } \\
\text { flux seen by an isotropic } \\
\text { detector. It is propor- } \\
\text { tional to the photon } \\
\text { density. }\end{array}$ \\
\hline
\end{tabular}
depends in various ways on the energy and direction of the radiation and is represented by the $f_{n}$ 's. A few examples are:

The numerical results of this paper pertain to flux type (d). By this choice they become comparable to the results of an extensive set of calculations for infinite media by the moment-method [5]. 


\subsection{Monte Carlo Estimates}

In the evaluation of expression (2) for the flux, the sum over collisons must be cut off for $n>N$, and the integration over the intermediate energies and angles is carried out by the Monte Carlo method. A suitable value of the cutoff $N$ depends on the absorbing properties of the medium, the depth of penetration, and the spectral-energy range over which one wants to know the flux. Calculations for an infinite medium show that for all practical purposes the energy flux vanishes below $30 \mathrm{kev}$ in water, below $50 \mathrm{kev}$ in iron, $75 \mathrm{kev}$ in tin, and $100 \mathrm{kev}$ in lead, for source energies between 0.5 and $10 \mathrm{Mev}$. The photon energy histories were therefore terminated at these limits. Trial calculations indicated that under these circumstances, for a barrier thickness up to 16 mean free paths $\left(\mu_{0} t=16\right)$, it was sufficient to let $\mathrm{N}=25$ for water, 12 for iron, 9 for tin, and 6 for lead.

Energy-angle histories of length $N$ were sampled according to the distribution function $Q_{N}$. The detailed equations for doing this are well known. They are presented elsewhere, together with the adaptations required or desirable for computations on an automatic computer [6].

Let $h_{n j}$ be an initial portion of length $n \leq N$ of the $j$ th sample history $h_{N j}$, and let $P_{n j}(z, t)$ be the corresponding crossing-probability. If a sample of $J$ histories is available, the flux of radiation in a specified spectral interval $R_{E}$ and angular interval $R_{\theta}$ can be estimated as follows:

$$
\begin{aligned}
\int_{R_{E}} d E \int_{R_{\theta}} d \theta F(E, \theta, z, t)= & \frac{K}{J} \sum_{j=1}^{J} \sum_{n=0}^{N} \\
& f_{n j} P_{n j}(z, t) \Delta_{1}\left(E_{n j}\right) \Delta_{2}\left(\theta_{n j}\right),
\end{aligned}
$$$$
\text { where }
$$

$$
\begin{aligned}
& \Delta_{1}\left(E_{n j}\right)\left\{\begin{array}{l}
=1, \text { if } E_{n j} \text { is in the interval } R_{E} \\
=0, \text { if } E_{n j} \text { is outside the interval } R_{E}
\end{array}\right. \\
& \Delta_{2}\left(\theta_{n j}\right)\left\{\begin{array}{l}
=1, \text { if } \theta_{n j} \text { is in the interval } R_{\theta} \\
=0, \text { if } \theta_{n j} \text { is outside the interval } R_{\theta} .
\end{array}\right.
\end{aligned}
$$

If one wants to know the value of the flux for fixed values of $E$ and $\theta$ rather than in intervals, the sampling procedure must be modified so as to be conditional not only on the initial photon state but also on the final state $E_{n}=E$ and $\theta_{n}=\theta$. For this purpose one replaces $P_{n}$ by $P_{n}^{*}\left(h_{n}^{*}\right) \Psi\left(E_{n-1}, \theta_{n-1} ; E, \theta\right)$, where

$$
h_{n}^{*}=\left\{\begin{array}{c}
E_{0}, E_{1}, \ldots, E_{n-1}, E \\
\theta_{0}, \theta_{1}, \ldots, \theta_{n-1}, \theta
\end{array}\right\} .
$$

We have been content to obtain the flux in energy and angular intervals because the main body of calculations was done in any case for the total intensity integrated over all spectral energies and directions.

In conformity with the usage that has become common in shielding work, the results for the intensity are presented in the form of buildup factors $B(z, t)$, which are equal to the ratio of the total to the unscattered energy flux:

$$
B(z, t)=\frac{\int_{0}^{E_{0}} d E \int_{0}^{\pi} d \theta F(E, \theta, z, t)}{K f_{0} P_{0}} .
$$

The Monte Carlo estimates of the buildup factors are the mean values

$$
\bar{B}(z, t)=(1 / J) \sum_{j=1}^{J} B_{j}(z, t),
$$

where

$$
B_{j}(z, t)=\left(E_{0}\left|\sec \theta_{0}\right| P_{0}\right)^{-1} \sum_{n=0}^{N} E_{n j}\left|\sec \theta_{n j}\right| P_{n j}(z, t) .
$$

The standard deviation of the buildup factor estimate is $J^{-1 / 2} \sigma(z, t)$ where

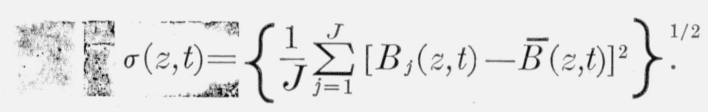

The buildup factors (and their standard deviations) were estimated from the same set of energyangle histories for three specific situations:

(i) $\bar{B}(0, t)=$ energy reflection buildup factor ${ }^{3}$

(ii) $\bar{B}(t, t)=$ energy transmission buildup factor

(iii) $\bar{B}(t, \infty)=$ energy buildup factor for the flux at depth $t$ within a semi-infinite medium.

\subsection{Correlation}

The difference between $\bar{B}(t, t)$ and $\bar{B}(t, \infty)$ is predominantly due to photons with histories such that in an infinite medium they would cross the plane $z=t$, have their directions reversed as the result of one or a few collisions in the vicinity of $t$, and would then make another crossing. Thus $\bar{B}(t, \infty)$ isloosely speaking - a function of two factors: a probability $p_{1}(t)$ of penetration to depth $t$, and a probability $p_{2}$ of being turned around in the vicinity of the $t$-plane. On the other hand, $\bar{B}(t, t)$ only contains the factor $p_{1}(t)$. The greatest statistical fluctuations in a Monte Carlo calculation are found in the estimate of $p_{1}(t)$, because only a small part of the sampled histories provides information concerning deep penetration. If the same set of energy-angle histories is used for the estimate of $\bar{B}(t, \infty)$ and $\bar{B}(t, t)$, it is to be expected that the estimate of the ratio of these two quantities will have a much smaller statistical dispersion than the estimates of the individual buildup factors because the ratio no longer contains the troublesome factor $p_{1}(t)$. Unscattered radiation should be excluded from the comparison because it

The energy reflection buildup factor has thel ollowing physica! significance. Consider an isotropic detector placed in the path of a broad beam of radiation, and denote the energy flow through the detector by $F_{0}$. Then place a barrier of thickness $t$ immediately behind the detector. This increases the energy flow through the detector to $F$. Then $\bar{B}(0, t)=F / F_{0}$. The quantity $\bar{B}(0, t)-1$ is related to but not identical with the energy albedo, which as commonly defined pertains to but not identical w 
s not affected by the boundaries. We therefore subtract 1 from both buildup factors and introduce the ratio

$$
k(t)=\frac{\bar{B}(t, t)-1}{\bar{B}(t, \infty)-1} .
$$

A Monte Carlo estimate of $k$ has an estimated standard deviation $J^{-1 / 2} \sigma_{k}$, where

$$
\begin{gathered}
\sigma_{k}=k\left\{\left[\frac{\sigma(t, t)}{\bar{B}(t, t)-1}\right]^{2}+\left[\frac{\sigma(t, \infty)}{\bar{B}(t, \infty)-1}\right]^{2}-\right. \\
\left.2 \rho \frac{\sigma(t, t)}{\bar{B}(t, t)-1} \frac{\sigma(t, \infty)}{\bar{B}(t, \infty)-1}\right\}^{1 / 2},
\end{gathered}
$$

and

$$
\rho(t)=\frac{(1 / \mathcal{J}) \sum_{j=1}^{J}\left[B_{j}(t, t)-\bar{B}(t, t)\right]\left[B_{j}(t, \infty)-\bar{B}(t, \infty)\right]}{\sigma(t, t) \sigma(t, \infty)}
$$

is the correlation coefficient of $\bar{B}(t, t)$ and $\bar{B}(t, \infty)$. The two buildup factors have a strong positive correlation so that $\rho \approx 1$ and the value of $\sigma_{k} / k$ is small. For moderate and deep penetrations, $\bar{B}(t, \infty)$ is practically identical with the buildup factor for an infinite medium which can be obtained by a reliable moment-calculation. These known results, together with an estimate of the ratio $k(t)$, provide an accurate indirect method for determining the transmission buildup factor which requires much smaller sample sizes than a direct estimate. Thus the Monte Carlo and moment-methods complement each other: one provides knowledge of the boundary effect, the other knowledge of deep penetration of radiation. In section 5 the advantage of this correlation technique is demonstrated.

\section{Solution of the Trarsport Equation for the Crossing-Probabilities}

The crossing-probabilities obey the following integral equations:

$P_{n+1}(z)=\frac{c\left(E_{n}\right)}{\left|\cos \theta_{n}\right|} \int_{0}^{z} d z^{\prime} P_{n}\left(z^{\prime}\right) e^{-\left[\mu_{A}\left(E_{n+1}\right)+c\left(E_{n+1}\right)\right]\left(z-z^{\prime}\right) / \cos \theta_{n+1}}$

if $\cos \theta_{n+1}>0$.

$P_{n+1}(z)=\frac{c\left(E_{n}\right)}{\left|\cos \theta_{n}\right|} \int_{z}^{t} d z^{\prime} P_{n}\left(z^{\prime}\right) e^{-\left[\mu_{A}\left(E_{n+1}\right)+c\left(E_{n+1}\right)\right]\left(z-z^{\prime}\right) / \cos \theta_{n+1}}$

if $\cos \theta_{n+1}<0$.

$$
P_{0}(z)=e^{-\left[\mu_{A}\left(E_{0}\right)+c\left(E_{0}\right)\right] z / \cos \theta_{0}} .
$$

These equations express the fact that the probability of passage through the z-plane after $n+1$ collisions is equal to the probability of passage through the plane $z^{\prime}$ after $n$ collisions, times the probability of a collision between $z^{\prime}$ and $z^{\prime}+d z^{\prime}$, times the probability of passage from $z^{\prime}$ to $z$ without further scattering or absorption, the triple product being averaged over all values of $z^{\prime}$ compatible with the boundary conditions.

We shall find it convenient to introduce in place of the probabilities $P_{n}$ the related quantities

$$
G_{n}(z)=\left|\sec \theta_{n}\right| P_{n}(z)
$$

which already incorporate the trigonometrical factor required for the type of flux which we want to determine. The following abbreviated notation will be adopted:

$$
\left.\begin{array}{l}
\mu_{n}=\mu_{A}\left(E_{n}\right)+c\left(E_{n}\right) \\
c_{n}=c\left(E_{n}\right) \\
\eta_{n}=\cos \theta_{n} \\
u_{n}=0 \text { if } \cos \theta_{n}>0 \\
u_{n}=t \text { if } \cos \theta_{n}<0
\end{array}\right\}
$$

Equations (10a, b, and c) can then be rewritten in the form

$$
\begin{gathered}
G_{n+1}(z)=\frac{c_{n}}{\eta_{n+1}} \int_{u_{n+1}}^{z} d z^{\prime} G_{n}\left(z^{\prime}\right) e^{-\frac{\mu_{n+1}}{\eta_{n+1}}\left(z-z^{\prime}\right)} \\
G_{0}(z)=\frac{1}{\eta_{0}} e^{-\frac{\mu_{0}}{\eta_{0}} z}
\end{gathered}
$$

To solve these equations, assume that $G_{n}$ can be expressed in the form

$$
G_{n}(z)=\sum_{m=0}^{n} A_{m}^{n} e^{-\frac{\mu_{m}}{\eta_{m}}\left(z-u_{m}\right)} .
$$

Substitution of eq (14) into (13a) yields the equation

$$
\begin{aligned}
G_{n+1}(z)= & \frac{c_{n}}{\eta_{n+1}} \sum_{m=0}^{n} \frac{A_{m}^{n} \eta_{m}}{\eta_{n+1} \mu_{m}-\eta_{m} \mu_{n+1}} \\
& \left\{e^{-\frac{\mu_{m}}{\eta_{m}}\left(u_{n+1}-u_{m}\right)} e^{-\frac{\mu_{n+1}}{\eta_{n+1}}\left(z-u_{n+1}\right)}-e^{-\frac{\mu_{m}}{\eta_{m}}\left(z-u_{m}\right)}\right\}
\end{aligned}
$$

so that the functional form of $G_{n+1}$ is the same as that of $G_{n}$. The expansion coefficients are

$$
\begin{aligned}
& A_{m}^{n+1}=\frac{c_{n}}{\eta_{n+1}} \frac{1}{\frac{\mu_{n+1}}{\eta_{n+1}}-\frac{\mu_{m}}{\eta_{m}}} A_{m}^{n} \text { for } 0 \leq m \leq n \\
& A_{n+1}^{n+1}=-\sum_{m=0}^{n} A_{m}^{n+1} e^{-\frac{\mu_{m}}{\eta_{m}}\left(u_{n+1}-u_{m}\right)} .
\end{aligned}
$$

We have thus obtained inductively the general solution for $G_{n}(z)$. Although the solution is rather formal and not readily surveyed, it is convenient for numerical evaluation with an automatic computer because of its recursive nature. 
It is possible that $\eta_{m} \mu_{n+1}-\eta_{n+1} \mu_{m}=0$, so that singularities occur in the recursion relations $(16 \mathrm{a}, \mathrm{b})$. But this difficulty is apparent rather than real, because upon substitution of the coefficients $A_{m}^{n+1}$ into the expression for $G_{n+1}$ the singularities occur pairwise and cancel each other. This can be seen by a reexamination of (15). Note that the existence of the singularity implies that $\eta_{n+1} \eta_{m}>0$, because both $\mu_{m}$ and $\mu_{n+1}$ are positive. It follows that $u_{m}$ is equal to $u_{n+1}$ : Therefore, the factor multiplying: $A_{m}^{n}$ in eq (15) is

$$
\alpha_{m}^{n}=\frac{c_{n} e^{-\frac{\mu_{n+1}}{\eta_{n+1}}\left(z-u_{n+1}\right)}}{\frac{\mu_{n+1}}{\eta_{n+1}}-\frac{\mu_{m}}{\eta_{m}}}\left\{e^{\left(\frac{\mu_{n+1}}{\eta_{n+1}}-\frac{\mu_{m}}{\eta_{m}}\right)\left(z-u_{n+1}\right)}-1\right\} .
$$

As $\eta_{m} \mu_{n+1}-\eta_{n+1} \mu_{m} \rightarrow 0$

$$
\alpha_{m}^{n} \rightarrow c_{n}\left(z-u_{m}\right) e^{-\frac{\mu_{m}}{\eta_{m}}\left(z-u_{m}\right)} .
$$

If the singularities are removed by a limiting procedure, the functional form of $G_{n+1}$ is thus changed. For the numerical computation on an automatic computer we have found it simplest not to use the limiting procedure, but to stick to the unmodified formal solution, while eliminating singular cases by introducing a cutoff for the quantity $\alpha=\left(\mu_{n+1} / \eta_{n+1}\right)-$ $\left(\mu_{m} / \eta_{m}\right)$ in the denominator of eq (16a). Every $\alpha<10^{-3}$ was replaced by $10^{-3}$. The error incuired by this approximation increases with $\left|z-u_{m}\right|$. But even for deep penetration it is quite small. For example, when $\alpha\left(z-u_{m}\right)=20$, $[\exp (-0.001 \times 20)-1] /$ $10^{-3}=20.201$, so that the error is only 1 percent.

\section{Machine Computation}

The calculation was programed for the NBS automatic computer (SEAC). It was carried out in two stages. First, energy-angle histories (in groups of approximately 100) were generated by random sampling and stored on magnetic wire or tape. These histories were then used as input data for the second stage, in which the quantities $G_{n}$ (proportional to the crossing-probabilities $P_{n}$ ) were calculated. Any desired type of flux or buildup factor can be obtained by a suitable linear combination of the $G_{n}^{\prime}$ 's, the selection being made by an appropriate output code.

The buildup factor code provides for the computation of $\bar{B}(0, t), \bar{B}(t, t)$, and $\bar{B}(t, \infty)$, for $\mu_{0} t=2^{n} d$ $(n=0,1, \ldots, 5)$, d being a disposable parameter. Either all 18 or any subgroup of these buildup factors can be computed in an integrated operation. The standard deviations $\sigma(0, t), \sigma(t, t)$, and $\sigma(t, \infty)$, as well as the correlation coefficient $\rho(t)$ are also contained in the output.

Most of the computational effort was devoted to the buildup factors, but limited use was also made of output codes for energy spectra and angular distributions.

The amount of SEAC computing time depends on the number $N$ of collisions through which one follows the photon histories. As has been mentioned, it was found sufficient to let $N=25$ for water, 12 for iron, 9 for tin, and 6 for lead. The amount of machine time (seconds per history) required for the generation and storage of photon energy-angle histories was

$$
t_{1}=0.4 N \text {. }
$$

For the joint computation of 18 buildup factors, the machine time (seconds per history and buildup factor)

$$
t_{2}=0.18 \mathrm{~N}+0.0205 \mathrm{~N}^{2}
$$

for $6 \leq N \leq 25$. For the computation of an individual buildup factor, the machine time (seconds per history)

$$
t_{3}=1.3 \mathrm{~N} .
$$

It can be seen that the joint computation of several buildup factors was more economical.

A major difficulty in setting up the machine program was the scaling problem. SEAC operates only on numbers less than 4 in absolute value. But the coefficients $A_{m}^{n}$ in the expansion for $G_{n}$ can occasionally become extremely large because of sampling accidents. This difficulty was aggravated by the fact that singularities were not removed analytically from the recursion relations $(16 \mathrm{a}, \mathrm{b})$ but by a numerical cutoff procedure. This resulted in the occurrence of pairs of coefficients with approximately the same large absolute value, but opposite sign, whose weighted difference must be calculated with great accuracy. This required the use of floatingdecimal-point routines, which, although they were specially adapted to the problem, proved to be costly in computation time.

The occurrence of large terms with alternating signs in the series for $G_{n}$ provided a convenient check on the code and the computer operation. Clearly $G_{n}$, being a positive multiple of a probability, must be a positive quantity. It was found by experience that because of the alternating character of the series for $G_{n}$, almost any sort of code or machine trouble quickly led to the occurrence of negative $G_{n}$ 's. The machine was instructed to interrupt the computation whenever this happened. As many $G_{n}$ 's are computed per second, the absence of such interruptions for prolonged periods was a reasonable guarantee that the operation of the computer was error free.

\section{Results}

\subsection{Statistical Efficiency}

Before presenting the main body of our results, we shall discuss the statistical accuracy of the Monte Carlo estimates in detail for a sample calculation based on 300 photon histories, pertaining to $1-\mathrm{Mev}$ radiation incident normally on an iron barrier. In table 1 are listed, in addition to the estimated buildup factors and buildup factor ratios, other quantities of statistical interest including fractional standard deviations and correlation coefficients. 
TABLE 1. Statistical analysis of buildup factor estimates for 1-Mev radiation incident on an iron barrier

\begin{tabular}{|c|c|c|c|c|c|c|c|c|c|c|}
\hline$\mu_{0} t$ & $\bar{B}(0, t)$ & $\frac{\sigma(0, t)}{\bar{B}(0, t)-1}$ & $\bar{B}(t, t)$ & $\frac{\sigma(t, t)}{\bar{B}(t, t)-1}$ & $\bar{B}(t, \infty)$ & $\frac{\sigma(t, \infty)}{\bar{B}(t, \infty)-1}$ & $1-\rho$ & $k$ & $\frac{\sigma_{k}}{k}$ & $q$ \\
\hline $\begin{array}{l}0.5 \\
1.0 \\
2.0\end{array}$ & $\begin{array}{l}\text { 1. } 048 \\
\text { 1. } 057 \\
\text { 1. } 061\end{array}$ & $\begin{array}{r}0.0586 \\
.0696 \\
.0747\end{array}$ & $\begin{array}{l}\text { 1. } 40 \\
\text { 1. } 71 \\
\text { 2. } 43\end{array}$ & $\begin{array}{r}0.61 \\
.73 \\
1.00\end{array}$ & $\begin{array}{l}\text { 1. } 53 \\
\text { 1. } 89 \\
\text { 2. } 68\end{array}$ & $\begin{array}{r}0.43 \\
.58 \\
.89\end{array}$ & $\begin{array}{l}\text { 9. } 2 \times 10^{-2} \\
\text { 2. } 2 \times 10^{-2} \\
\text { 6. } 5 \times 10^{-3}\end{array}$ & $\begin{array}{r}0.755 \\
.798 \\
.851\end{array}$ & $\begin{array}{r}0.29 \\
.20 \\
.15\end{array}$ & $\begin{array}{r}0.4 \\
.3 \\
15.2\end{array}$ \\
\hline $\begin{array}{r}\text { 4. } 0 \\
8.0 \\
16.0\end{array}$ & $\begin{array}{l}\text { 1. } 061 \\
\text { 1. } 061 \\
\text { 1. } 061\end{array}$ & $\begin{array}{l}0747 \\
0747 \\
.0747\end{array}$ & $\begin{array}{r}\text { 4. } 07 \\
\text { 7. } 80 \\
\text { 17. } 8\end{array}$ & $\begin{array}{l}\text { 1. } 58 \\
\text { 2. } 90 \\
4.87\end{array}$ & $\begin{array}{r}4.45 \\
8.60 \\
20.0\end{array}$ & $\begin{array}{l}\text { 1. } 48 \\
\text { 2. } 68 \\
\text { 4. } 58\end{array}$ & $\begin{array}{r}\text { 4. } 9 \times 10^{-3} \\
3.4 \times 10^{-3} \\
1.85 \times 10^{-3}\end{array}$ & $\begin{array}{l}.890 \\
.895 \\
.884\end{array}$ & $\begin{array}{l}.18 \\
.31 \\
.41\end{array}$ & $\begin{array}{r}43.6 \\
67.2 \\
115.0\end{array}$ \\
\hline
\end{tabular}

Now let us see what the large amount of analysis introduced into the Monte Carlo calculation has accomplished. To estimate the transmission buildup factor for a barrier with thickness $\mu_{0} t=16$ with a standard deviation amounting to 5 percent of the buildup factor would require on the order of $10^{8}$ photon histories in an ordinary random sampling calculation. But according to table 1 the semianalytical method requires only $8.5 \times 10^{3}$ histories. Taking into account the estimated increase in computation time per history required in the semianalytical calculation, one finds that the effective reduction factor is $\approx 2,500$. This is respectable but not quite good enough for a computer with the capabilities of SEAC. But one can do better still through the use of correlated sampling, i. e., by estimating the buildup factor ratio $k(t)$. It can be seen from table 1 that, in accordance with expectations, the correlation coefficient $\rho$ is very close to unity, and the fractional standard deviation $\sigma_{k} / k$ correspondingly small. A 5 percent standard deviation now only requires a sample of 67 histories. Taking into account the increased computation time per history, the reduction of effort is 80 -fold. The over-all improvement factor is therefore $2,500 \times 80=200,000$.

The last column of table 1 lists the factor $q=$ $[\sigma(t, t) / \bar{B}(t, t)]^{2} /\left[\sigma_{k} / k\right]^{2}$, by which the correlated sampling technique reduced the required sample size. Statistical analyses at other energies and for other scattering media yield over-all improvements in efficiency of about the same magnitude.

\subsection{Buildup Factors}

Reflection buildup factors for semi-infinite media are listed in table 2 , a, for diverse conditions. The sample sizes were such that the quantities $\bar{B}(0, t)-1$, i. e., the increase of the flux due to reflection, had a statistical accuracy of 5 percent. It can be seen that energy reflection is appreciable only for low energies and low- $Z$ materials. Table $2, b$, shows the dependence of reflection on the barrier thickness for some typical cases. The outstanding feature of these results is the rapidity with which the reflection of energy assumes its maximum value. A barrier with a thickness of one, or at most two mean free paths of
TABLE 2. Energy reflection

a. Energy Reflection Buildup Factors for Semi-infinite Barriers

\begin{tabular}{|c|c|c|c|c|c|c|c|c|}
\hline \multirow{3}{*}{ Energy } & \multicolumn{2}{|c|}{ Water } & \multicolumn{2}{|c|}{ Iron } & \multicolumn{2}{|c|}{ Tin } & \multicolumn{2}{|c|}{ Lead } \\
\hline & \multicolumn{8}{|c|}{$e_{0}$} \\
\hline & $0^{\circ}$ & $60^{\circ}$ & $0^{\circ}$ & $60^{\circ}$ & $0^{\circ}$ & $60^{\circ}$ & $0^{\circ}$ & $60^{\circ}$ \\
\hline Mev. & & & & & & & & \\
\hline $\begin{array}{c}0.4 \\
.66\end{array}$ & $\begin{array}{l}\text { 1. } 240 \\
1.148\end{array}$ & $\begin{array}{l}\text { 1. } 278 \\
\text { 1. } 189\end{array}$ & $-\cdots$ &..- & -- & -. & - & - \\
\hline 1. 0 & 1.081 & $\begin{array}{l}1.153 \\
\end{array}$ & 1.061 & 1.142 & 1.022 & 1.086 & 1.009 & 1. 042 \\
\hline $\begin{array}{l}\text { 4. } 0 \\
\text { S }\end{array}$ & 1.012 & $\begin{array}{l}1.035 \\
1 .\end{array}$ & 1.011 & - & 1.005 & 100 & 1.001 & $\ldots$ \\
\hline 10.0 & 1.002 & 1.007 & 1.01 & - & 1.000 & -1 & - & $\ldots$ \\
\hline
\end{tabular}

b. Dependence of Reflection on Barrier Thickness a

\begin{tabular}{|c|c|c|c|c|c|}
\hline \multirow{2}{*}{ Material } & \multirow{2}{*}{ Energy } & \multirow{2}{*}{$\theta_{0}$} & \multicolumn{3}{|c|}{$\mu_{0} t$} \\
\hline & & & 0.5 & 1 & 2 \\
\hline $\begin{array}{l}\text { Water } \\
\text { Do } \\
\text { Iron } \\
\text { Do } \\
\text { Tin } \\
\text { Lead }\end{array}$ & $\begin{array}{l}\text { Mev. } \\
0.66 \\
.66 \\
1.0 \\
1.0 \\
1.0 \\
1.0\end{array}$ & $\begin{array}{r}0^{\circ} \\
60^{\circ} \\
0^{\circ} \\
60^{\circ} \\
0^{\circ} \\
0^{\circ}\end{array}$ & $\begin{array}{l}0.65 \\
.61 \\
.79 \\
.89 \\
.95 \\
.97\end{array}$ & $\begin{array}{r}0.88 \\
.96 \\
.93 \\
.98 \\
.99 \\
1.00\end{array}$ & $\begin{array}{l}0.99 \\
1.00 \\
1.00 \\
1.00 \\
1.00 \\
1.00\end{array}$ \\
\hline
\end{tabular}

a Columns 4, 5 , and 6 give the values of $k(0)=[\bar{B}(0, t)-1] /[\bar{B}(0, \infty)-1]$.

$k(0)$ is a measure of the relative amount of reflected radiation from a finite barrier and from a semi-infinite medium.

the source radiation is already equivalent to a semiinfinite medium.

Table 3 contains an extensive list of buildup factor ratios $k(t)$, for water at $0.66,1$, and $4 \mathrm{Mev}$, for iron, tin, and lead at 1, 4, and $10 \mathrm{Mev}$. Again, it can be seen that the boundary effect, indicated by deviations of $k(t)$ from unity, is appreciable only at low energies and for low- $Z$ materials. It is significant that the ratios stay constant, within the limits of statistical error, as the barrier thickness is increased from $\mu_{0} t=4$ to $\mu_{0} t=16$. The physical reason for this constancy is the establishment of radiative near-equilibria in regions far from the source, which is well known from the diffusion theory for infinite media. Sufficiently deep in the material, both the energy spectrum and angular distribution of the radiation flux become very slowly varying functions of the penetration depth. Under these circumstances it is not surprising that the boundary effect, which depends on the characteristics of these distributions, likewise remains practically unchanged. 
TABLE 3. Buildup factor ratio $k(t)=[\bar{B}(t, t)-1] /[\bar{B}(t, \infty)-1]$ for comparing transmission of radiation through a finite barrier with penetration to an equal depth in a semi-infinite medium

\begin{tabular}{|c|c|c|c|c|c|c|c|}
\hline \multirow{2}{*}{ Material } & \multirow{2}{*}{ Energy } & \multicolumn{6}{|c|}{$\mu_{0} t$} \\
\hline & & a 1.0 & 1.0 & 2.0 & 4.0 & 8.0 & 16.0 \\
\hline Water & $\left\{\begin{array}{l}\text { Mev } \\
0.66 \\
1 \\
4\end{array}\right.$ & $\begin{array}{r}0.601 \\
.661 \\
.849\end{array}$ & $\begin{array}{r}0.663 \\
.720 \\
.885\end{array}$ & $\begin{array}{r}0.713 \\
.754 \\
.912\end{array}$ & $\begin{array}{r}0.783 \\
.821 \\
.920\end{array}$ & $\begin{array}{r}0.785 \\
.828 \\
.926\end{array}$ & $\begin{array}{r}0.784 \\
.830 \\
.933\end{array}$ \\
\hline Iron & $\left\{\begin{array}{r}1 \\
4 \\
10\end{array}\right.$ & $\begin{array}{l}.790 \\
.890 \\
.941\end{array}$ & $\begin{array}{l}.798 \\
.910 \\
.959\end{array}$ & $\begin{array}{l}.851 \\
.923 \\
.972\end{array}$ & $\begin{array}{l}.890 \\
.936 \\
.974\end{array}$ & $\begin{array}{r}.895 \\
.932 \\
.978\end{array}$ & $\begin{array}{l}.894 \\
.949 \\
.977\end{array}$ \\
\hline Tin.. & $\left\{\begin{array}{r}1 \\
4 \\
10\end{array}\right.$ & $\begin{array}{l}.889 \\
.941 \\
.951\end{array}$ & $\begin{array}{l}.911 \\
.926 \\
.960\end{array}$ & $\begin{array}{l}.924 \\
.955 \\
.962\end{array}$ & $\begin{array}{l}.935 \\
.967 \\
.973\end{array}$ & $\begin{array}{l}.938 \\
.974 \\
.971\end{array}$ & $\begin{array}{l}.946 \\
.978 \\
.969\end{array}$ \\
\hline Lead.... & $\left\{\begin{array}{r}1 \\
4 \\
10\end{array}\right.$ & $\begin{array}{l}.939 \\
.941 \\
.986\end{array}$ & $\begin{array}{l}.951 \\
.977 \\
.990\end{array}$ & $\begin{array}{l}.969 \\
.982 \\
.995\end{array}$ & $\begin{array}{l}.975 \\
.990 \\
.992\end{array}$ & $\begin{array}{l}.979 \\
.992 \\
.994\end{array}$ & $\begin{array}{l}.982 \\
.994 \\
.995\end{array}$ \\
\hline $\begin{array}{r}\text { Estimated } \\
\text { accuracy } \\
(\%)\end{array}$ & $\cdots$ & \pm 5.0 & \pm 2.0 & \pm 1.5 & \pm 1.5 & \pm 2.0 & \pm 2.5 \\
\hline
\end{tabular}

a Comparison of a barrier with an infinite medium.

TABLE 4. Energy-transmission buildup factors

\begin{tabular}{|c|c|c|c|c|c|c|c|}
\hline \multirow{2}{*}{ Material } & \multirow{2}{*}{$\begin{array}{c}\text { Energy } \\
\text { Mev }\end{array}$} & \multicolumn{6}{|c|}{$\mu_{0} t$} \\
\hline & & 0.5 & 1.0 & 2.0 & 4.0 & 8.0 & 16.0 \\
\hline Water & $\left\{\begin{array}{l}0.66 \\
1 \\
4\end{array}\right.$ & $\begin{array}{l}\text { 1. } 49 \\
\text { 1. } 40 \\
\text { 1. } 22\end{array}$ & $\begin{array}{l}\text { 1. } 96 \\
\text { 1. } 80 \\
\text { 1. } 42\end{array}$ & $\begin{array}{l}\text { 3. } 10 \\
\text { 2. } 72 \\
\text { 1. } 83\end{array}$ & $\begin{array}{l}\text { 5. } 99 \\
5.01 \\
\text { 2. } 60\end{array}$ & $\begin{array}{c}13.3 \\
10.5 \\
4.21\end{array}$ & $\begin{array}{c}39.4 \\
25.7 \\
7.20\end{array}$ \\
\hline Iron & $\left\{\begin{array}{r}1 \\
4 \\
10\end{array}\right.$ & $\begin{array}{l}1.40 \\
1.20 \\
1.07\end{array}$ & $\begin{array}{l}\text { 1. } 72 \\
\text { 1. } 36 \\
1.16\end{array}$ & $\begin{array}{l}\text { 2. } 43 \\
\text { 1. } 72 \\
\text { 1. } 35\end{array}$ & $\begin{array}{l}4.07 \\
2.50 \\
1.75\end{array}$ & $\begin{array}{l}7.80 \\
4.17 \\
2.80\end{array}$ & $\begin{array}{c}17.8 \\
7.45 \\
5.85\end{array}$ \\
\hline Tin & $\left\{\begin{array}{r}1 \\
4 \\
10\end{array}\right.$ & $\begin{array}{l}1.29 \\
1.16 \\
1.06\end{array}$ & $\begin{array}{l}\text { 1. } 56 \\
\text { 1. } 31 \\
\text { 1. } 12\end{array}$ & $\begin{array}{l}\text { 2. } 10 \\
\text { 1. } 63 \\
\text { 1. } 26\end{array}$ & $\begin{array}{l}3.15 \\
2.35 \\
1.59\end{array}$ & $\begin{array}{l}\text { 5. } 31 \\
\text { 4. } 12 \\
\text { 2. } 75\end{array}$ & $\begin{array}{c}10.2 \\
9.41 \\
8.22\end{array}$ \\
\hline Lead.- & $\left\{\begin{array}{c}1 \\
4 \\
10\end{array}\right.$ & $\begin{array}{l}1.20 \\
1.11 \\
1.03\end{array}$ & $\begin{array}{l}1.35 \\
\text { 1. } 23 \\
1.08\end{array}$ & $\begin{array}{l}\text { 1. } 63 \\
\text { 1. } 44 \\
\text { 1. } 17\end{array}$ & $\begin{array}{l}2.09 \\
1.98 \\
1.40\end{array}$ & $\begin{array}{l}2.87 \\
3.28 \\
2.17\end{array}$ & $\begin{array}{l}4.24 \\
7.46 \\
6.47\end{array}$ \\
\hline
\end{tabular}

The results of table 3 pertain to a comparison of finite barriers with a semi-infinite medium. For distances $\mu_{0} t \geq 4$, a comparison with an infinite medium would yield practically identical results. Column 3 shows the buildup factor ratios for $\mu_{0} t=1$ obtained by a Monte Carlo calculation for transmission and a moment-calculation for an infinite medium. It can be seen that these ratios are up to 10 percent lower than the corresponding ratios for a semi-infinite medium. For $\mu_{0} t=2$ there is a similar but extremely small reduction, but our data are insufficient for us to quote a value of the reduction.

Table 4 lists the transmission buildup factors for the same range of conditions as in table 3 . For $\mu_{0} t=0.5,1$, and 2 , the results were obtained entirely by the Monte Carlo calculation. For greater barrier thicknesses the values quoted are the result of applying the correction factors of table 3 to an extensive set of calculations for an infinite medium [5]. The infinite-medium results are stated to be accurate to within 5 percent. The accuracy of the derived transmission buildup factors is therefore of the order of 6 to 7 percent. The direct Monte Carlo results for shallow penetration have about the same accuracy.

\subsection{Application of the Monte Carlo Method to an Infinite-Medium Problem}

For beavy elements the boundary effect is so small that the Monte Carlo method does not add much new knowledge. Yet it can be utilized as a convenient and laborsaving tool for the extension of available results. As an example of such an application we have considered the penetration of radiation from a plane monodirectional oblique source in an infinite medium. This problem can be handled by the moment-method, but requires much more work than the corresponding problem for plane monodirectional sources with zero obliquity, or plane isotropic sources. We considered a 4-Mev plane oblique source in lead. A set of 100 energyangle histories were selected that yielded buildup factors in close agreement with those of a momentcalculation for a monodirectional zero obliquity source for penetrations up to $\mu_{0} t=16$. Then we performed a rotation of the angular part of these histories. Provision for doing this is built into the SEAC code for the computation of the $G_{n}$ 's. After rotation, these histories can then be used as input data for oblique source problems, and the results can be expected to be reliable, since the energy part of the bistories, known to be properly representative,

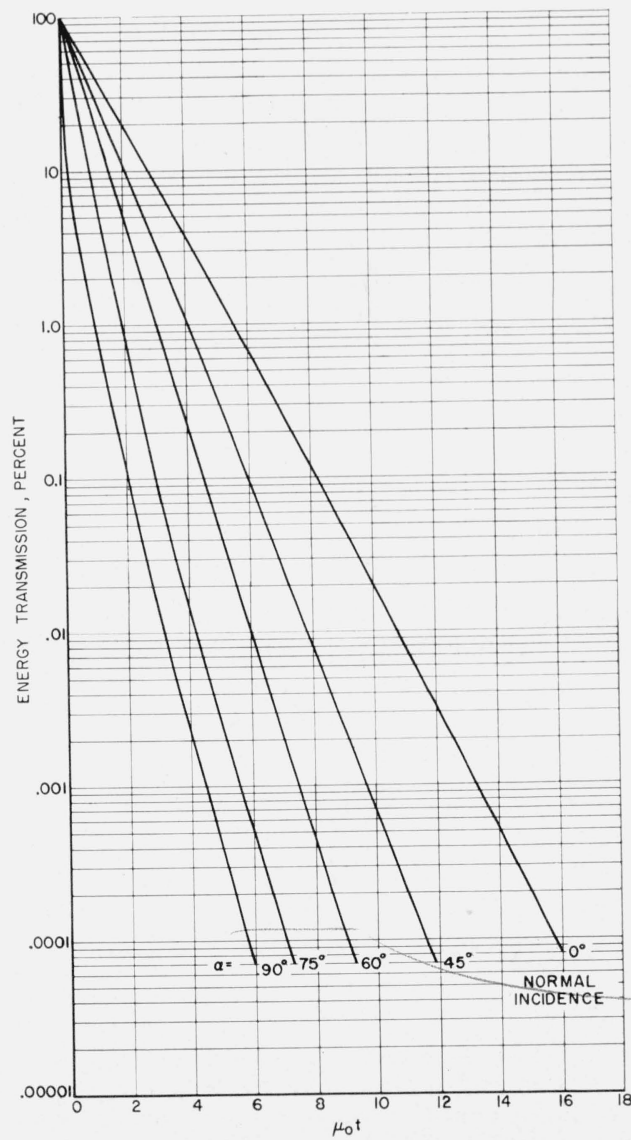

FIgURE 2. Transmission of radiation from a 4-Mev plane monodirectional oblique source through a lead barrier. 
remained unchanged by the rotation. In this manner we have made quick calculations for obliquity angles $\theta_{0}=45^{\circ}, 60^{\circ}, 75^{\circ}$, and $90^{\circ}$. As a final check, the fluxes from sources with different obliquities were summed so as to obtain the flux from a plane isotropic source. Again the agreement with a direct moment-calculation was close. Thus a fix on the accuracy of the Monte Carlo results was obtained at two points, and in spite of the small sample size the penetration of radiation to very great depths could be calculated with an accuracy which we estimate to be 5 to 7 percent. The results of the calculation are shown in figure 2 in the form of plots of the percent-energy-transmission (=buildup factor $\left.\times \exp \left(-\mu_{0} t\right)\right)$ versus $\mu_{0} t$, for different obliquity angles.

\subsection{Energy Spectra and Angular Distributions}

In figure 3 we show for normally incident 0.66 Mev radiation the energy spectra of the scattered flux transmitted through water barriers with thicknesses $\mu_{0} t=4$ and $\mu_{0} t=8$. The corresponding energy spectra at the same depths in an infinite medium are also shown. (The histograms pertain to the Monte Carlo results, the curves to a momentcalculation.) The shaded areas between corresponding histograms for transmission and an infinite medium are a measure of the boundary effect. It can be seen that the presence of boundaries removes a large part of the low-energy end of the spectrum, but leaves spectral components near the source energy unchanged. It is noteworthy that the spectra are quite similar at both depths. This lends support to our argument that the constancy of the buildup factor ratios is connected with the occurrence of radiative equilibria.

Figure 4 pertains to the same conditions as figure 3 , and shows the angular distribution of the scattered transmitted energy flux, and of the corresponding flux in an infinite medium. The shaded areas again indicate the boundary effect. The shaded areas

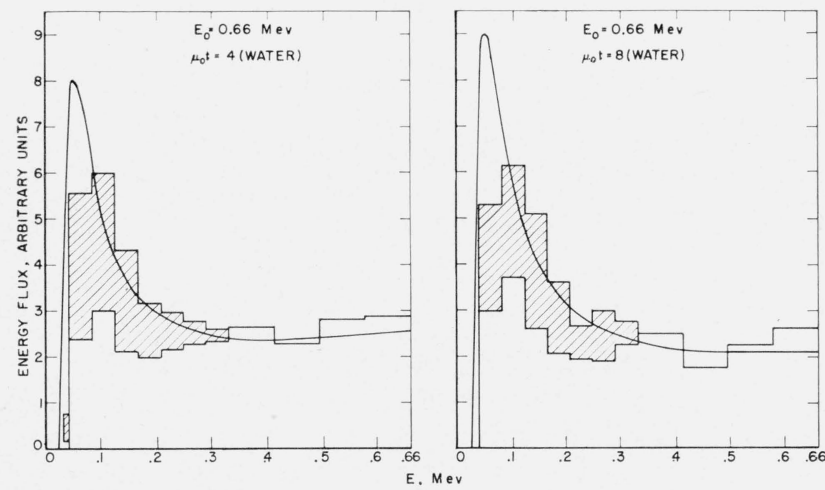

FIGURE 3. Energy spectra of scattered radiation transmitted through water barriers of thickness $\mu_{0} t=4$ and $\mu_{0} t=8$, for normally incident beams of $0.66-$ Mev photons.

The unshaded parts of the histograms pertain to penetration through a finite barrier, while the shaded areas indicate the additional radiation that would penetrate to a depth $t$ in an infinitely extended medium. The curves accompanying the histograms represent the results of calculations by the moment-method for an infinite medium. for negative values of $\cos \theta$ represent photons which crossed the boundary $2,4,6, \cdots$ times, while the areas for positive values of $\cos \theta$ represent photons which crossed the boundary $3,5,7, \cdots$ times in an infinite medium. The relatively small number of photons in the latter group indicates that repeated crossings of any plane deep in an infinite medium are quite unlikely, so that it should be possible to calculate the boundary effect in reasonable approximation by infinite-medium theory, by setting the transmitted flux equal to the infinite-medium flux integrated over positive values of $\cos \theta$. The angular distributions are similar to the energy spectra in that they do not change their shape as

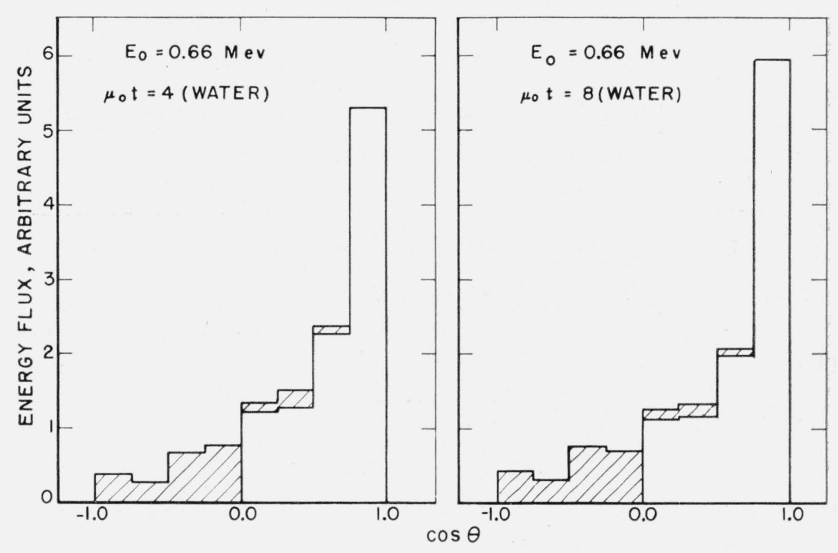

Figure 4. Angular distributions of scattered radiation transmitted through water barriers of thickness $\mu_{0} t=4$ and $\mu_{0} !=8$, for normally incident beams of 0.66-Mev photons.

The unshaded parts of the histograms pertain to penetration through a finite barrier, while the shaded areas indicate the additional radiation that would penetrate to a depth $t$ in an infinitely extended medium. The angle $\theta$ measures the direction of the transmitted radiation with respect to the normal to the barrier face. Shaded areas for positive values of $\cos \theta$ represent photons that would cross the boundry plane at least three times in an infinite medium.
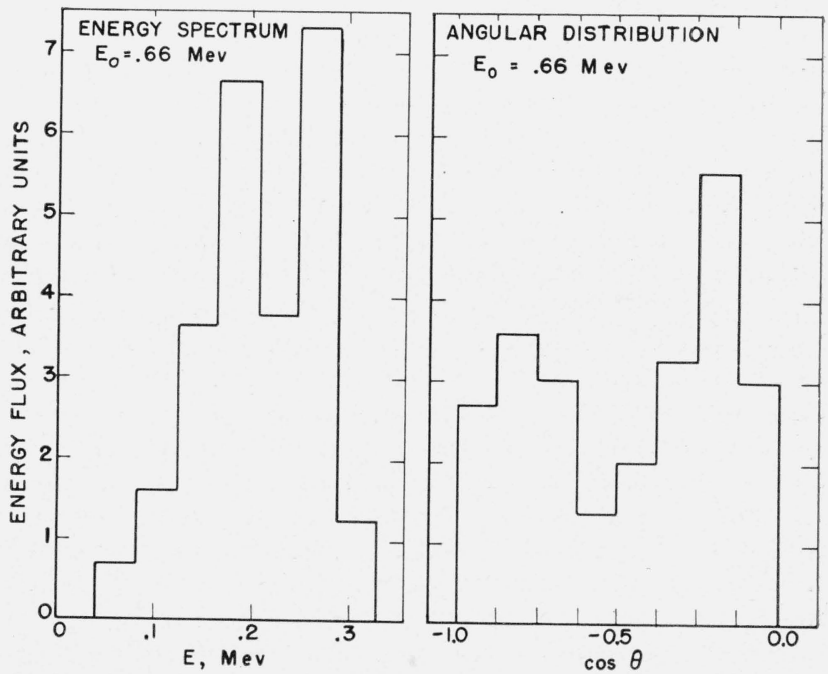

FIgURE 5. Energy spectrum and angular distribution of radiation reflected from a semi-infinite water barrier, for a normally incident beam of $0.66-\mathrm{Mev}$ photons.

The angle $\theta$ indicates the direction between the direction of the reflected photons and the normal to the barrier face. 
the barrier thickness is increased from $\mu_{0} t=4$ to $\mu_{0} t=8$.

Figure 5 shows the angular and energy distributions of reflected radiation due to $0.66-\mathrm{Mev}$ photons incident normally on a semi-infinite water barrier. Both distributions have two distinct peaks. Their locations are such that they appear to imply the existence of two preferred modes of reflection, one associated with a first scattering through approximately $180^{\circ}$, the other with a first scattering through approximately $90^{\circ}$.

\section{Discussion}

\subsection{Comparison with Experiments}

A recent survey of experiments on the penetration and diffusion of $\gamma$-radiation [5] indicates that few experiments have been done for plane source radiation that are comparable with our calculations. Moreover, the uncertainty of the experimental results is usually at least of the order of magnitude of the boundary effect. It would seem that the boundary effect could best be checked by a differential experiment in which one measures the flux through a detector, both with and without backing material behind the detector.

The penetration of radiation with energies between 0.66 and $2.76 \mathrm{Mev}$ through iron slabs has been measured by Beach et al. [7]. Their experimental values agree to within a few percent with the buildup factors listed in table 4. But the experimental results include large corrections for air-scattering, so that the evidence is not as conclusive as one might wish.

Kirn, Kennedy, and Wyckoff [8] measured the penetration of $\mathrm{Co}^{60}$ radiation (1.17 and $1.33 \mathrm{Mev}$ ) through concrete barriers. An attempt has been made to match their results by a calculation for an aluminum barrier with a concrete-equivalent electron density. (The photoelectric absorption cross section per electron is very nearly the same for concrete as for aluminum, except at extremely low energies.) The penetration in an infinite aluminum medium was found by interpolation from calculations of Goldstein and Wilkins [5]. These results were then corrected for the boundary effect with the use of table 3 of this paper. Finally, the predicted buildup factors were increased by small amounts (not more than $2 \%$ ) to account for the energy response of the experimental detector. One more major adjustment must be made prior to a comparison of theory and experiment. The experimental cobalt source was $1 \mathrm{~cm}$ thick, so that there was an appreciable chance for Compton scattering and resultant energy degradation of the radiation in the source. The degraded radiation is absorbed much more strongly than the primary radiation while passing through the concrete barrier. When considering the attenuation at moderate and great depths one should renormalize the source strength so as to include only undegraded photons emerging from the source. An approximate single-scattering calculation leads to the estimate that the effective source strength is $.82 \pm .05$ times the nominal source strength. Table 5 shows the experimental and theoretical energy transmission (for normal incidence of the $\mathrm{Co}^{60}$ radiation on the concrete barrier). The agreement with the adjusted experimental values is good. Although the source strength correction constitutes a weakness of the com arison (at least insofar as verification of the boundary effect is concerned), it is significant that by adjusting a single normalization constant one can get agreement between theory and experiment over a wide range of barrier thicknesses.

\section{TABLE 5. Energy transmission by a concrete barrier}

Comparison of the experimental transmission found by Kirn et al. [8], and the predicted transmission. (Transmission=buildup factor $\left.\times \exp \left(-\mu_{0} t\right).\right)$

\begin{tabular}{|c|c|c|c|c|}
\hline & \multicolumn{4}{|c|}{$\begin{array}{l}\text { Energy transmission, in percent, } \\
\text { with concrete barrier thickness of }\end{array}$} \\
\hline & $5.0 \mathrm{in.}$ & $10.0 \mathrm{in.}$ & 15.0 in. & 17.5 in. \\
\hline Experiment, uncorrected & 39.0 & 11. 0 & 2. 80 & 1.40 \\
\hline Experiment, corrected for the deg- & & & & \\
\hline $\begin{array}{l}\text { radation of radiation in the source. } \\
\text { Theory }\end{array}$ & $\begin{array}{l}46.8 \\
44.3\end{array}$ & $\begin{array}{l}13.2 \\
13.7\end{array}$ & $\begin{array}{l}\text { 3. } 36 \\
\text { 3. } 41\end{array}$ & $\begin{array}{l}1.68 \\
1.69\end{array}$ \\
\hline
\end{tabular}

The experimental literature on the reflection of gamma radiation from barriers is even scarcer than that on transmission. We have learned of some unpublished measurements by Kirn, Kennedy, and Wyckoff ${ }^{4}$ of the reflection of $\mathrm{Co}^{60}$ radiation from effectively semi-infinite concrete and lead barriers. (The lead barrier actually was a concrete block fronted by $1 / 4$ in. of lead, but this combination was in effect equivalent to a solid lead barrier.) By interpolation based on the results in table 2 we obtained reflection buildup factors which, according to the comparison in table 6 , are in reasonably good agreement with the experimental values.

ТАВLE 6. Energy-reflection buildup factors for $\mathrm{Co}^{60}$ radiation incident on semi-infinite barriers

Comparison of the theory with the experiment of Kirn et al.

\begin{tabular}{|c|c|c|c|}
\hline Material & $\begin{array}{c}\text { Angle of } \\
\text { incidence, } \theta_{0}\end{array}$ & Experiment & Theory \\
\hline $\begin{array}{l}\text { Concrete } \\
\text { Lead. }\end{array}$ & $\left\{\begin{array}{r}\text { deg } \\
0 \\
60 \\
0 \\
60\end{array}\right.$ & $\begin{array}{l}1.063 \\
1.086 \\
1.012 \\
1.041\end{array}$ & $\begin{array}{l}\text { 1. } 065 \\
1.110 \\
1.0095 \\
1.040\end{array}$ \\
\hline
\end{tabular}

\subsection{Comparison With Other Calculations}

In table 7 a comparison is made for buildup factors for shallow and moderate penetration depths, pertaining to $0.66 \mathrm{Mev}$ radiation incident on water barriers. The comparison includes the results of this paper, infinite-medium calculations by the moment-method [10] and the results of two other Monte Carlo calculations, one of them done by a direct stochastic analog method [9], the other

${ }^{4}$ H. O. Wyckoff, private communication. 
TABLE 7. Comparison of buildup factors obtained by various methods for 0.66-Mev radiation in water medium

\begin{tabular}{|c|c|c|c|c|c|c|c|c|}
\hline Method & \multicolumn{3}{|c|}{$\begin{array}{c}\text { Transmission, } \\
\bar{B}(t, t)\end{array}$} & \multicolumn{3}{|c|}{$\begin{array}{c}\text { Penetration in } \\
\text { semi-infinite } \\
\text { medium, } \\
\bar{B}(t, \infty)\end{array}$} & \multicolumn{2}{|c|}{$\begin{array}{l}\text { Reflection } \\
\text { from semi- } \\
\text { infinite } \\
\text { medium, } \\
\bar{B}(0, \infty)\end{array}$} \\
\hline & \multicolumn{8}{|c|}{$\mu_{0} t$} \\
\hline & 1 & 2 & 4 & 1 & 2 & 4 & $\theta_{0}=0$ & $\begin{array}{l}\theta_{0}= \\
60^{\circ}\end{array}$ \\
\hline $\begin{array}{l}\text { Monte Carlo (this } \\
\text { paper) }\end{array}$ & \multirow{5}{*}{$\begin{array}{l}1.96 \\
1.89 \\
1.92\end{array}$} & \multirow{4}{*}{$\begin{array}{l}3.10 \\
3.21 \\
3.30\end{array}$} & \multirow{4}{*}{$\begin{array}{l}5.99 \\
5.64 \\
5.55\end{array}$} & \multirow{4}{*}{2.48} & \multirow{4}{*}{$\begin{array}{l}4.02 \\
4.07 \\
\text { 3. } 75\end{array}$} & \multirow{4}{*}{$\begin{array}{l}7.15 \\
6.98 \\
6.55\end{array}$} & \multirow{4}{*}{$\begin{array}{l}1.148 \\
1.15 \\
1.14\end{array}$} & \multirow{4}{*}{$\begin{array}{l}1.189 \\
1.18 \\
-\ldots\end{array}$} \\
\hline Monte Carlo (NBS) & & & & & & & & \\
\hline Monte Carlo (NRL) & & & & & & & & \\
\hline [9] & & & & & & & & \\
\hline ment-method $[10]$ & & & & & & & & - \\
\hline
\end{tabular}

based on an analytical integration over a sampled collision density [6]. The agreement of the various methods is on the whole satisfactory.

\subsection{Comments}

We have demonstrated the constancy of the boundary effect for thick barriers, and the resultant lack of necessity to do deep-penetration Monte Carlo calculations, only for plane geometry. But it appears plausible that a similar situation will also prevail in other geometries since the constancy of the boundary effect appears to be rooted in the occurrence of equilibrium spectra far from a radiation source.

The advantage of incorporating knowledge obtained from other sources into a Monte Carlo calculation through the technique of correlated sampling has been clearly demonstrated. In fact, it was only this technique which made it possible for us to survey the boundary effect for a variety of conditions without excessive computation.

The analytical treatment of the space variable likewise proved successful, but we feel that it may have been too much of a good thing. From the standpoint of computing efficiency, there is an optimum amount of analysis that should be put into a Monte Carlo calculation, which depends on the nature of the problem and the capabilities of the computing machine. It is questionable whether we operated near this optimum. If one is interested in the radiation intensity, it is not unreasonable to take, as we have done, relatively small samples and to analyze them exhaustively. But if energy spectra and angular distributions are desired, it is probably prefarable to use larger and more representative samples of energy-angle histories and to analyze them less thoroughly. One possible compromise would be to confine the analytic treatment of the space variable to a limited number of collisions, and then to revert to a stochastic calculation.

The authors thank Anne Futterman for aid and advice in programing the problem for SEAC.

\section{References}

[1] L. V. Spencer and U. Fano, J. Research NBS 46, 446 (1951) RP2213.

[2] L. V. Spencer, Phys, Rev. 88, 793 (1952).

[3] G. H. Peebles, Rand Report R-240 (1952); J. Appl. Phys. 24, 1272, 1437 (1953).

[4] H. Kahn, Nucleonics 6, No. 6, 60 (1950); Applied Mathematics Series NBS (June 1951) AMS12.

[5] H. Goldstein and J. E. Wilkins, Jr., AEC Report NYO 3075 (June 1954).

[6] M. J. Berger, Reflection and transmission of $\gamma$-radiation by barriers: Monte Carlo ealculation by the collision density method, J. Research NBS 55, 343 (1955) RP2640.

[7] L. A. Beach, R. B. Theus, and W. R. Faust, Phys. Rev. 92, 355 (1953).

18] F. S. Kirn, R. J. Kennedy, and H. O. Wyckoff, Radiology 63, 94 (1954).

[9] L. A. Beach, R. B. Theus, J. D. Plawchan, and W. R. Faust, NRL Report 4412 (April 1954).

[10] M. J. Berger, The penetration of $\gamma$-radiation from a plane monodirectional oblique source, J. Research NBS $\mathbf{5 6}$, (1956) (in press).

Washington, September 1, 1955. 